\title{
ADDRESSING THE MULTICHANNEL SELECTION, SCHEDULING AND COORDINATION COST
}

\author{
Mthulisi Velempini ${ }^{1}$ and Mqhele E. Dlodlo ${ }^{2}$ \\ ${ }^{1}$ University of Limpopo, South Africa \\ ${ }^{2}$ University of Cape Town, South Africa
}

\begin{abstract}
We discuss a new multichannel coordination and scheduling challenge called the Multichannel Scheduling Cost (MSC). It is caused by the inability of the control channel to schedule simultaneous transmissions resulting in data channels lying idle and their bandwidth underutilized. As a result, wasted bandwidth increases as the number of data channels increases. To address this challenge, a cyclic scheduling Algorithm (CSA) is proposed. It employs one dedicated signaling channel and 4 data channels. It is premised on a proactive channel reservation scheme which reduces the idleness of channels. The scheme ensures that data channels are reserved while they are still busy. The data channels are reserved while their remaining transmission duration is equal to the virtual carrier sensing duration. This gives the next pair sufficient time to reserve the current data channel before it becomes available, limiting the MSC to the first cycle. The simulation results show a performance benefit of the CSA scheme in addressing the effects of the MSC.
\end{abstract}

Keywords: Bandwidth Utilization; Connectivity; Control channel; Multichannel Scheduling Cost

\subsection{Introduction}

The implementation of multichannel MAC protocols in Wireless Mesh Networks (WMN) has generated some encouraging results, which increases network capacity and throughput. However, there are still outstanding challenges which degrade the capacity of multichannel networks. For example, the channel assignment, selection and coordination strategies result in the creation of a new multichannel degradation challenge we call the Multichannel Scheduling Cost (MSC). MSC wastes the capacity of data channels during the reservation phase. Data channels remain idle for long durations waiting for their turns to be reserved, resulting in the underutilization of their capacity. The amount of wasted bandwidth increases linearly as the number of data channels increases. Furthermore, the MSC challenge is recurring and repetitive. To address this challenge, we propose a proactive data channel reservation scheme which addresses the MSC and improves the utilization of data channels. The scheme reduces the idle durations of data channels while improving their capacity.

Data channels are reserved in phases. The first channel to be reserved in the first cycle suffers the least degradation caused by the MSC, while the last data channel suffers the worst degradation. The adverse effect of the MSC increases as the number of data channels increases. The MSC is a scheduling challenge is addressed in this paper through the implementation of a proactive scheduling scheme which limits the MSC to the first cycle, thereby improving the performance of multichannel MAC protocols. 
The MSC interference challenge affects the multichannel MAC protocols which implement control channel coordination schemes. Besides the perceived performance benefits of the control channel schemes, they give rise to the MSC. We characterize and demonstrate the impact of the MSC on network performance. A proposed MSC solution is presented and evaluated through network simulations. The results show an improvement in the performance of the network.

The scheme is implemented in a backhaul of static mesh routers equipped with intelligence. The word intelligence in this context means the ability of the network to acquire knowledge of the network status, to facilitate distributed decision-making, to aid data channel reservation. The number of channels was not changed in the simulation environment.

The paper is structured as follows: The next Section justifies the implementation of a common signaling channel. Section 3 discusses related work. The system model is discussed and analyzed in Section 4 and 5 respectively. The simulation environment is presented in Section 6 and the results are analyzed in Section 7. Section 8 summarizes the paper.

\subsection{Motivation}

Multichannel MAC schemes implementing a common control channel have been designed. The protocols show a significant improvement in network performance and they offer reasonable capacity. However, their implementations have given rise to a new multichannel scheduling cost. The MSC has not been addressed in literature. It is a challenge which degrades network performance.

The use of multi-channels avail more bandwidth which is a requirement of the next generation networks however, channel selection, access and coordination remains a challenge. Channel coordination challenges result in the degradation of bandwidth. This challenge is evident in MSC in which a number of channels remain idle while they wait for the active channel to finish its transmission. Furthermore, channels are accessed in turns, one at a time successively leading to repetitive cycles of bandwidth wastage. A scheduling algorithm which reduces either the idle duration of channels or which addresses these idle slots is required. In this paper, an algorithm which restricts the idleness of channel to the first cycle and eliminates them in the successive cycles is presented

\subsection{Related Work}

Multichannel MAC protocols which employ a single transceiver and a control channel, which is reserved for signaling, are examined. The impact of the MSC on network performance is highlighted. The emphasis is on the MSC challenge and how it degrades the capacity of multichannel MAC protocols.

The scheme in [1] employs a temporary control channel called the quiescent channel. The protocol makes use of a dedicated busy tone channel to coordinate data channels access to prevent data collision. The busy tone channel wastes bandwidth, though it does not give rise to the MSC challenge it requires optimization.

The second algorithm proposed in [1] which divides a communication session into control and data windows is subjected to the effects of the MSC. The MSC is experienced during the control window. Given that the control window precedes any communication, the effects of the MSC are recurring. Lastly, during the data window there is no manifestation of the MSC. All the channels are utilized during the data window. 
The protocol utilizes only one channel during the control window sessions. The data channels lie idle and their bandwidth is wasted. It allows more than one pair of nodes to reserve one data channel thereby worsening the effects of the MSC when nodes take turns to transmit on the reserved data channels.

In [2], a window based scheme is proposed. Data channels are reserved during ATIM window through the default channel. One data channel can be reserved by many nodes, which later contend to access it during the data window. The scheme is similar to the one described above however, the implementation of the virtual carrier sensing (VCS) results in the worst case of the MSC which is repetitive both during the ATIM and the data windows. The two forms of MSC are experienced degrading the network performance significantly.

A window based scheme which implements a temporary control channel is also proposed in [3]. The protocol consists of a contention reservation interval (CRI) and the contention free interval (CFI). Terminals contend for data channels during the CRI and then defer their transmissions until the start of the CFI. The deferment of transmission and the reservation of channels during the CRI give rise to the MSC challenge.

The protocol proposed in [4] implements the concept of home channels instead of a control channel. The implementation of the home channels addresses the MSC at the cost of partitioning the network and lack of reach.

In [5], a single transceiver Multichannel MAC protocol which implements contention and data windows is proposed. A three way channel reservation handshake is implemented with an additional control packet called the Multichannel CTS Recognition (MCTS-R). The increasing control packets worsen the effects of the MSC. Furthermore, the data channels are not used during the control window and they are reserved in phases sequentially resulting in the manifestation of the MSC challenge.

The Group Allocation Multi hop Multiple Access (GAMMA) proposed in [6] implements multiple transmission channels. Each transmitter has a unique channel which reduces the effects of the MSC. The process of realigning data slots, the management of group membership, registration and de-registration procedures however, worsens the MSC.

The protocol proposed in [7] introduces the idea of distributed information sharing to ensure that communicating pairs do not make independent decisions. The proposed protocol does implement a dedicated control channel however; it is optimized to solve the terminal deafness problem and collisions in the data channels. The protocol introduces up to six control packets which worsens the impact of the MSC which is repetitive.

In [8] a Distributed Queue Dual Channel (DQDC) scheme is proposed. The DQDC scheme introduces 4 control packets which increases the idle durations of the data channels which in turn worsens the impact of the MSC challenge.

In [9] a scheme which implements a control channel and $\mathrm{N}$ traffic channels is presented. A node first senses all the data channels including the control channel before it sends an RTS packet. Both the transmitter and the receiver sense all the channels which increases the MSC. The effects of the MSC have been worsened by an increase in the size of control packets.

In [10], an asynchronous Multichannel Coordination Protocol (AMCP) is proposed. The protocol employs a single transceiver and a dedicated common control channel. Bandwidth is wasted when the preferred channel of the sender is rejected by the receiver. The sender has to select randomly the receiver's suggested data channels and restarts the contention cycle. Furthermore, control 
packets have increased in size. In addition, a new control packet, the Reserve (RES) has been introduced. Bandwidth is further degraded as nodes attempt to reserve data channels more than once. All these configurations contribute to the worst case of the MSC challenge.

In [11], a busy tone scheme is proposed. The scheme assumes that nodes can transmit and receive simultaneously. A channel is divided into two sub channels, a data and a control channel. Busy tone signals are transmitted on the control channel. The data channels are not used during data channel reservation, which results in the MSC challenge.

In general, multichannel MAC protocols outperform single channel MAC protocols as demonstrated in [12], [13]. The multichannel schemes have been investigated in cognitive networks with a measure of success [14], [15]. They have also been implemented in vehicular ad hoc networks [16], in wireless sensor networks [17], [18], [19], [20] and in general ad hoc networks [21], [22], [23], [24], and [25]. This shows that multichannel technique is a promising approach requiring further investigation.

\subsection{System Model}

In this section, we discuss the architectural design ideal for the implementation of the MSC solution. The architecture is memory based which is equipped with nodes with sufficient network status to facilitate the reservation of data channels.

The architecture is equipped with a network of mesh routers deployed in the ad hoc network of mesh clients. The mesh routers are networked to facilitate communication within the network of mesh clients. The architecture is configured such that each mesh router is within the transmission range of the next mesh router. The mesh routers store network status information which is shared with mesh clients to support their medium access and scheduling decisions. The proposed architecture is depicted in Fig 1. The architecture consists of routers enclosed in blue lines. All the Mobile nodes are assumed to be within the coverage of the network of mesh routers. The network of support mesh routers provides a support layer to the mesh architecture.

The network support mesh routers maintain a data structure we call a Network Status Table (NST) which is updated using overheard control packets. The network support nodes are referred to as the Network Status Table Nodes (NSTN). The NSTNs store information on the availability of data channels, the list of in use data channels and the duration of their unavailable - the interphase time. A data channel is available when its remaining transmission time is equal to the VCS duration of the next pair. The remaining time is determined by a hold off duration - the interphase time technique which allows data channels to be reserved in a proactive manner. 


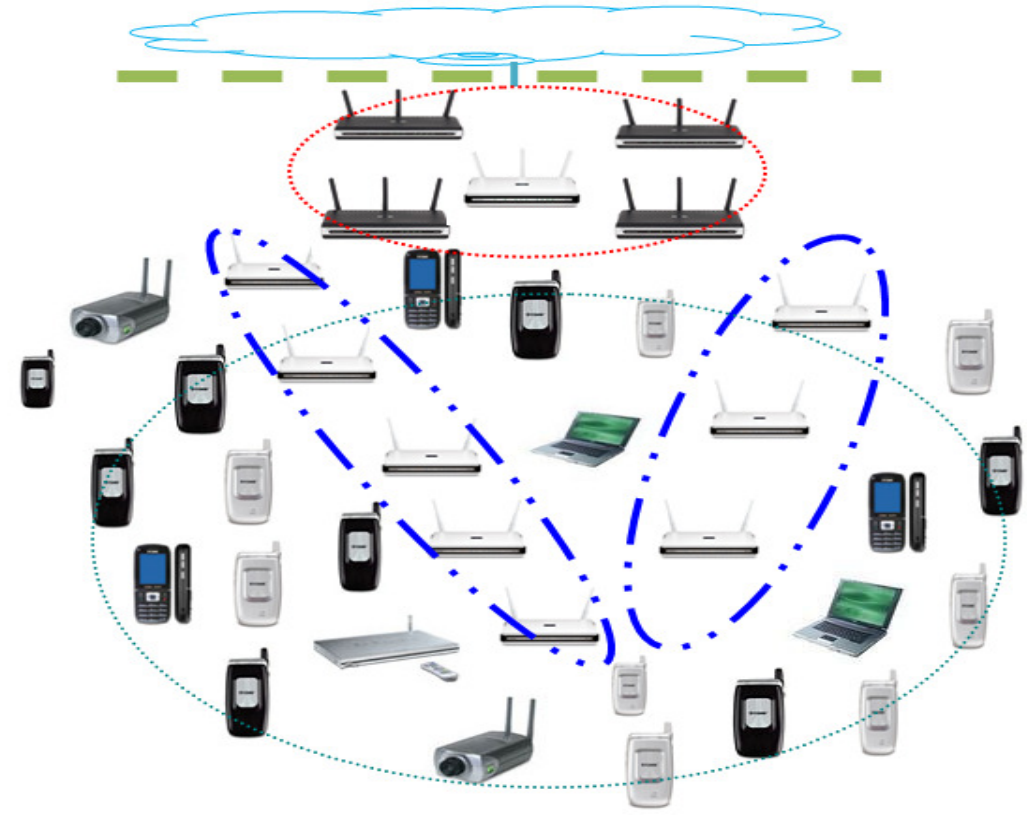

Fig 1. The architecture of the proposed network support infrastructure in a hybrid mesh network

A new inter-frame space known as the inter-phase replaces the DIFS ensures cyclical transmission of data. It is a proactive scheme which facilitates the reservation of data channels before they finish their current transmission, effectively reducing the idleness of data channels between the phases. The reduction of the idleness of data channels reduces the effects of the MSC. When data channels finish their transmission in the previous phase while waiting for their turn in the next phase to be reserved, they lie idle for long durations impacting negatively on the efficiency of the protocol which results in MSC. Given our proactive scheme, the wait period is reduced significantly which addresses the effects of the MSC.

A data channel is reserved when the inter-phase time has expired and nodes use the CSMA/CA to contend for the control channel. The inter-phase replaces the DIFS implemented in IEEE 802.11 MAC and its value is stored in the NAV of nodes. It also doubles as the transmission duration of data packets. The inter-phase time is a summation of data transmission duration plus one switching time minus the control channel handshake [26] [27]. The inter-phase time is stored in the NSTN as data transmission duration of the given data channel. The NSTNs maintain a sequence of the data channels to ensure that they are reserved in a round robin basis. Furthermore, the network status stored in tables is availed to mesh client node with insufficient knowledge of the status of the network. The said node probes the nearest NSTN. The maintenance of the network status tables is assigned to powerful nodes, the mesh routers. NSTN overhear control packets, use overhead data to update their data structures. The network support nodes are designed to equip mesh clients that cannot update their VANs after missing control packets.

Fig 2 demonstrates the functionality of the support scheme. The flow chart shows that depending on the availability of the network, a given node can contend for the control channel, otherwise it has to first probe the nearest NSTN for detailed network status. Three scenarios exist in which nodes may not have adequate knowledge of the network. These are the joining, the returning and start-up nodes.

A mesh client with insufficient network information probes the nearest NSTN before contending for the control channel to reserves a channel. Network related data is sent to the probing node by 
an NSTN. Overhearing nodes also update their tables; only if their tables are out dated. A node which listens on the control channel is expected to be up to date; hence there is no need for it to probe the NSTNs or to rely on probe responses.

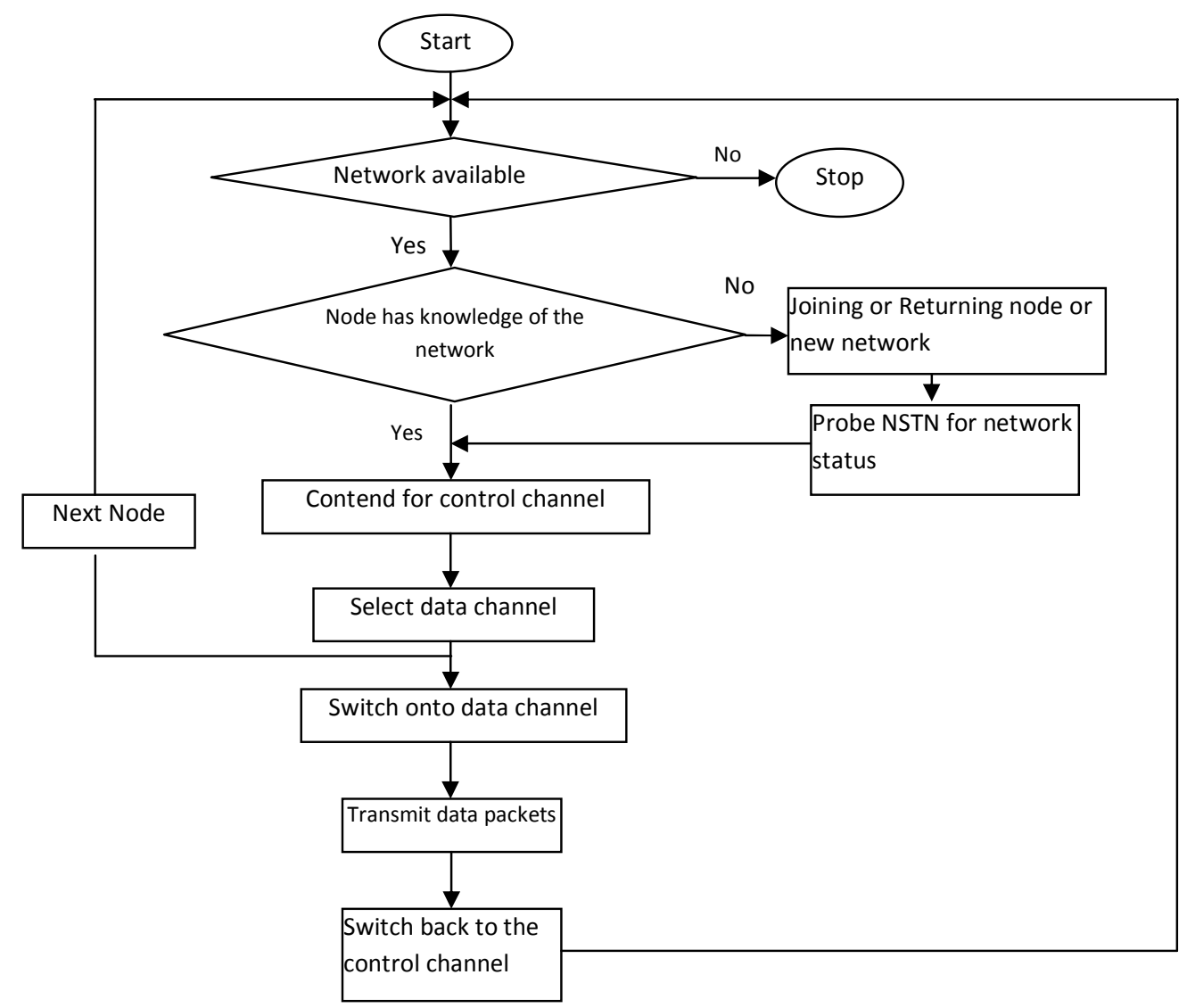

Fig 2. The implementation of the network infrastructure in the Cyclic Scheduling Algorithm

\subsection{Model Analysis}

The MSC wastes bandwidth on all the data channels and it is repetitive. However, the proposed scheme limits the MSC to the first cycle. This is achieved through the implementation of interphase time scheme. The data channels are reserved when they are still busy transmitting current data frames. The implementation of the inter-phase time ensures that data channels reservation is done proactively when data transmission is about to end. Secondly, the Extended Inter-Frame Space (EIFS) has been reconfigured and changed into a shorter Inter-Frame Space (IFS). In multichannel MAC protocols, there is no need for nodes which receive erroneous packets to defer for the EIFS duration. The EIFS worsens the MSC. The EIFS was designed to protect the ACK packets in single channel MAC protocols. In multichannel MAC protocols, ACK packets are transmitted on a different channel.

Fig 3 shows how the window based multichannel MAC protocols worsen the MSC. It also shows the extent at which data channels are underutilized during the control window. 


\begin{tabular}{|c|c|c|c|c|}
\hline Control Channel & Reservation & Data Frame & Reservation & Data Frame \\
\hline Data Channel 1 & DATA IDLE & Data Frame & DATA IDLE & Data Frame \\
\hline Data Channel 2 & DATA IDLE & Data Frame & DATA IDLE & Data Frame \\
\hline
\end{tabular}

Fig 3. The poor utilization of data channels in Window based multichannel MAC protocols.

The column is marked either as a control window or data window. The rows represent the channels and the top row represents the control channel. During the control window, the control channel is marked "Reservation" while in the data window it is marked "Data Frame". Data channels are reserved through the control channel during the control window and then as a data channel inside the data window to transmit data frames. The last two rows depict the two data channels. They are both marked "DATA IDLE" during the control window, demonstrating the underutilization of their capacity during the control window. It shows the extent to which the MSC affects the performance of the network.

Fig 4 characterizes and models the MSC. It also shows how the MSC challenge degrades the capacity of data channels. It shows the severity of the MSC and the extent at which control and data channels are underutilized. The vertical axis denotes the number of channels marked channel 0 to 7 . The horizontal axis denotes the elapsed transmission time. The control channel is assumed to be Channel 0 while the rest are data channels. The capacity of all data channels is underutilized between T0 and T1 while nodes are busy contending for the control channel and reserving Channel 1. Channel 2 is reserved between epoch T1 and T2, while all other channels lie idle except channel 1 which is currently transmitting data. In essence, Channel 1 is underutilized between T0 and T1, Channel 2, is underutilized between T0 and T2, Channel 3 is underutilized between $\mathrm{T} 0$ and T3, channel 4 between T0 and T4, channel 5 is underutilized between T0 and T5, while channel 6 is underutilized between T0 and T6. Lastly, channel 7 underutilized between T0 and $\mathrm{T} 7$.

As illustrated in Figure 4, the last channel to be reserved, channel 7 in this case is subjected to the worst effects of the MSC while the first channel (which is reserved first in phase) suffers the least degradation due to the effects of the MSC. On the other hand the control channel lies idle between T7 and T8. Thereafter the pattern is repeated for Data channels from T8 to T14. This shows that the effects of the MSC are repetitive and recurring. The MSC degrades severely the performance of the multichannel MAC protocols as illustrated in Fig 4. The illustration clearly demonstrates the significance of this work and the magnitude of the MSC challenge. The proposed model restricts the MSC to the first phase, there after its effects are eliminated. It is not possible to eliminate the MSC in the first phase, due to the fact that nodes cannot be reserved in a proactive manner in the first phase. Secondly, data transmissions cannot be scheduled to all the available data channels concurrently. Figure 5 characterize the MSC challenge. 


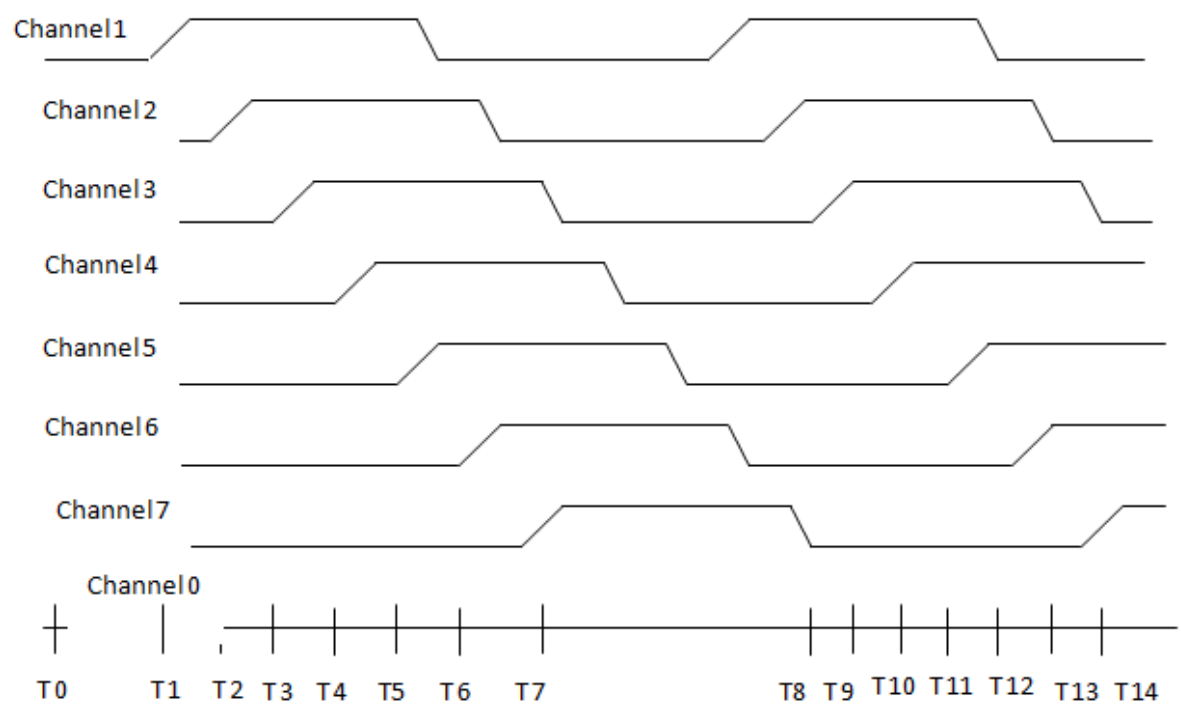

Fig 4. The Multichannel Scheduling Cost

Fig 5 depicts the behaviour of the MSC as more data channels are added. It also shows how the effects of the MSC increase with the increase in the number of channels. The 2 nd data channel incurs twice the degradation of the 1st data channel, while the degradation of the 3rd data channel is thrice that of the 1 st data channel. The degradation of the fourth data channel is 4 times that of the 1st data channel. Thus, the effects of MSC increase with the increase in the number of data channels. The total degradation of bandwidth is the summation of wasted bandwidth on all data channels. This is a high cost which degrades severely the performance of multi-channel MAC protocols. This cost requires an effective and efficient solution.

To calculate the wasted bandwidth due to the effects of the MSC, the following equations can be employed:

$$
\begin{gathered}
\mathrm{MSC}=\mathrm{wb}+2 \mathrm{xwb}+3 \mathrm{xwb}+\ldots \ldots+(\mathrm{n}-1) \times \mathrm{wb}+\mathrm{n} \times \mathrm{wb} \\
\operatorname{MSC}(x)=\sum_{n=1}^{n} \mathrm{n}(\mathrm{wb})
\end{gathered}
$$

Where $\mathrm{n}$ is the number of data channels and wb is the amount of wasted bandwidth. 


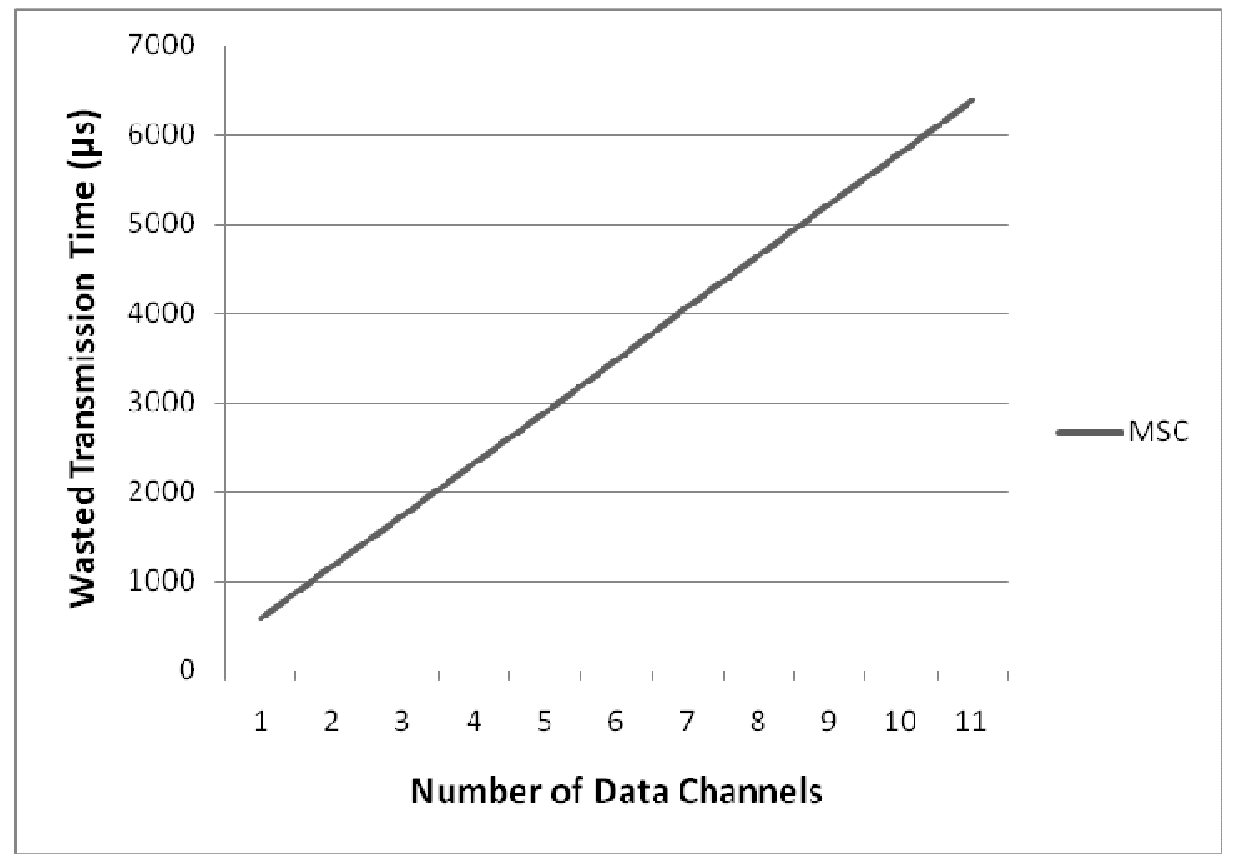

Fig 5. The Behaviour of the MSC as the channels increase in number

\subsection{Simulation Model}

The proposed scheme was implemented in NS 2. The performance of the scheme was evaluated in a multi-channel frame work developed in [10]. The EIFS was reconfigured and changed to shorter IFS called the CIFS. The CIFS is the summation of the transmission duration of the CTS packet and the SIFS duration. Nodes defer for CIFS instead of EIFS upon receiving erroneous control packets. In the proposed scheme, nodes defer for SIFS after the data channel has been reserved. The two channel switching times have been added to data packet transmission durations.

A proactive reservation strategy which reduces the MSC by improving the utilization of the channels is implemented. The CIFS, the data channel reservations strategy and the deferring rules are designed to reduce idle durations of channels. They improve the utilization of channels which improves the performance of the proposed protocol. The scalability of the scheme and its robustness in large networks with high incidents of interference was also evaluated.

A network with 5 channels, a control channel and 4 data channels was implemented. The channels were assumed to be orthogonal and of equal bandwidth, which was set to $2 \mathrm{Mbs}$. The number of channels was changed however; the size of the network was varied between 4 and 30 nodes to evaluate the scalability of the proposed scheme. A Non Ad Hoc (NOAH) routing algorithm was also implemented.

In the simulation, 5 different network sizes in terms of number of nodes were considered. The network sizes with the following number of nodes were considered: 4, 6, 8, 10, and 30 nodes. Simulations were run for each network size, and the topologies were assumed to be general. Data packets were assumed to be 1000 bytes long and of type CBR. A single hop network set up was assumed with packets sent directly to the receiver. The RTS and CTS packets were transmitted on the control channel and the DATA and ACK packets were transmitted on the data channels. We 
also took into account 2 channel switching times, each assumed to be $224 \mu \mathrm{s}$. For detailed simulation environment see the table below.

Table1. MAC Layer and other Parameter Settings

\begin{tabular}{|l|l|}
\hline Parameter & Value \\
\hline SIFS & $10 \mu \mathrm{s}$ \\
\hline DIFS & $50 \mu \mathrm{s}$ \\
\hline EIFS & $364 \mu \mathrm{s}$ \\
\hline CIFS & $56(\mu \mathrm{s})$ \\
\hline Slot time & $20 \mu \mathrm{s}$ \\
\hline Data rate & 2 Mbps \\
\hline Basic rate & 2 Mbps \\
\hline Control Channel & 1 \\
\hline Data Channels & 4 \\
\hline Switching time & $224 \mu \mathrm{s}$ \\
\hline Data Type & CBR \\
\hline Packet size & 1000 bytes \\
\hline CW max & 1023 \\
\hline CW min & 7 \\
\hline Short Retry Limit & 7 \\
\hline Long Retry Limit & 4 \\
\hline PLCP Length & 192 bits \\
\hline PLCP rate & 1 Mbps \\
\hline RTS & 20 bytes \\
\hline CTS & 14 bytes \\
\hline ACK & 14 bytes \\
\hline MAC Header - Data & 28 bytes \\
\hline MAC Header rate & Basic rate \\
\hline Routing Agent & NOAH \\
\hline Network Sizes & $4,6,8,10$ and 30 nodes \\
\hline PLCP Header & 6 Bytes \\
\hline Control Channel & $2 \mu \mathrm{s}$ \\
\hline Data Channel Propagation & $4 \mu \mathrm{s}$ \\
\hline PLCP Preamble & 18 Bytes \\
\hline & \\
\hline
\end{tabular}

\subsection{Simulation Results}

\subsection{Analysis of dropped packets}

In this segment, we analyze the number of dropped packets in evaluating the performance of the proposed scheme. The emphasis is on the number of dropped packets caused by longer system response times. The efficiency of service points is therefore investigated. In this case the service time of the control channel is examined. The services time affects the number of dropped packets 
from the buffer. This metric relates to the utilization of channels in the sense that, if the utilization of the channel is poor, longer system response times are expected, and more packets are dropped as a result. Improving the utilization of channels causes fewer packets to be dropped. The improvement in the utilization of the channel and the dropping of fewer packets is a sufficient indicator of the effectiveness of the proposed scheme in addressing the MSC. In Fig 7, the scheme is evaluated in a network with 4 nodes.

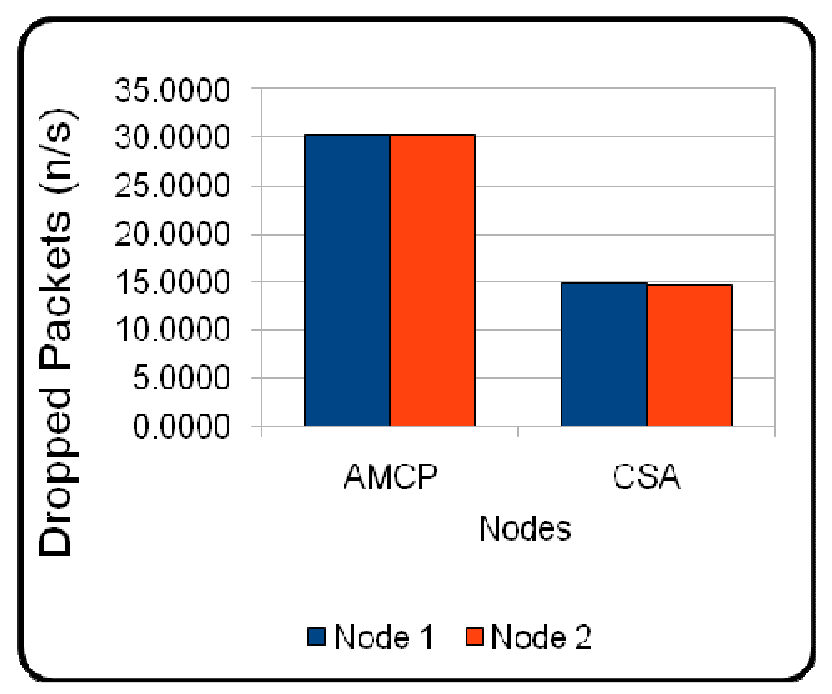

Fig 7. Analyzing dropped packets in a network with 4 nodes.

The CSA dropped the least number of packets demonstrating that the scheme did experience fewer and shorter delays as a result of improved channel utilization. The packets were not subjected to longer waiting durations before they were processed. In CSA, the next pair does not wait for the current pair to finish its transmission; it proactively reserves it during the course of the ongoing data transmission. The improved service rates in the CSA effectively address the effects of the MSC.

The nodes were increased to 6 in Fig 8. The nodes were grouped into three transmitters and three receivers, generating three data flows. The CSA scheme did drop fewer packets as compared to the AMCP. All the data flows dropped fewer packets, a clear indication that the proposed CSA scheme is effective in addressing the MSC.

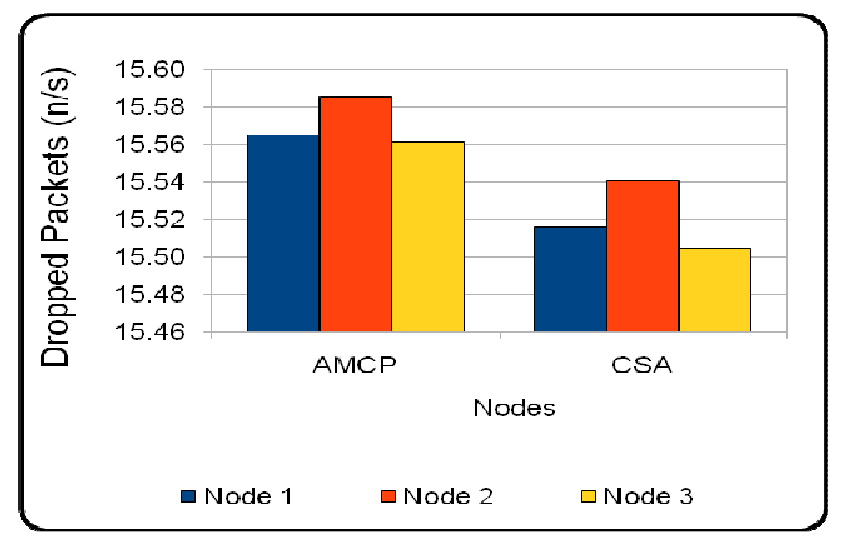

Fig 8. The performance of the CSA in a network with 6 nodes 
In Fig 9, the network size was scaled up. The number of nodes was increased to 10 . The number of data flows was more that the number of the data channels. As a result, the contending fifth data flow had an effect on the overall performance of the network. The two schemes did experience the highest number of dropped packets, which degraded the performance of the two schemes.

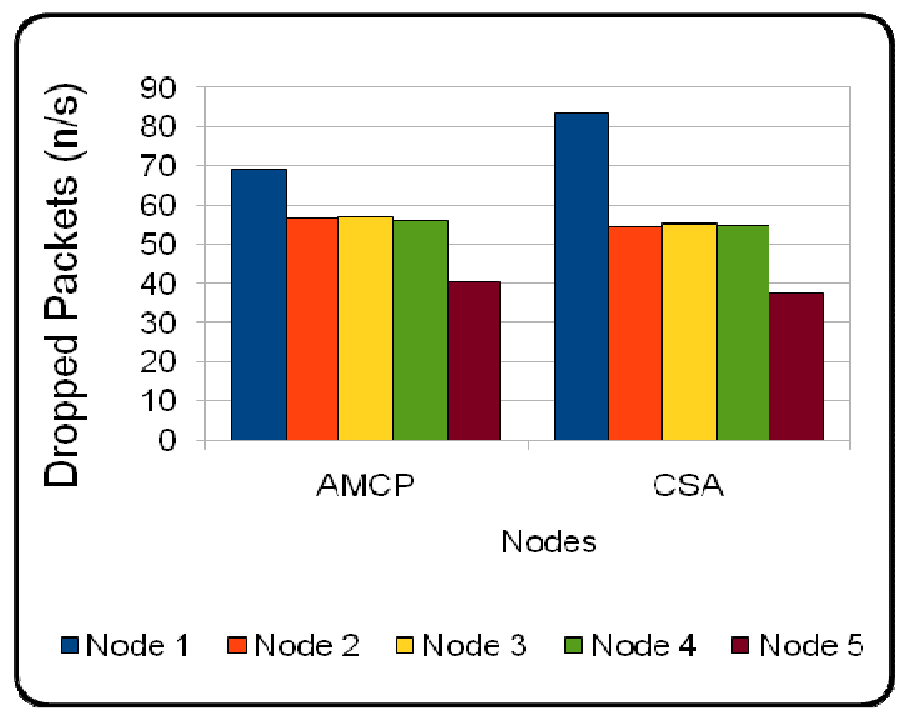

Fig 9. The performance of the CSA in a general network with 10 nodes

In the nutshell, the proposed scheme performed better than the AMCP. However, it was outperformed in the 1st data flow. This is due to its longer transmission durations and the effects of the interfering fifth data flow. The CSA is scalable and its performance improves as the network increase in size. This shows that the scheme addresses effectively the MSC challenge. The results in Fig 10 validate this argument.

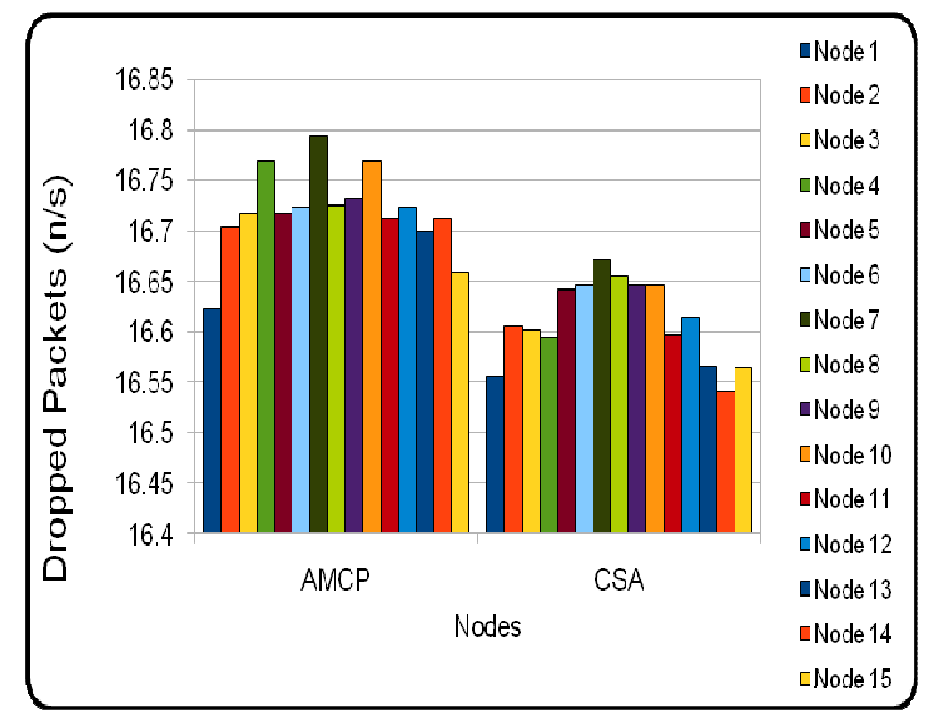

Fig 10. The total number of dropped packets in a network with 30 nodes

In Fig 10, the number of nodes was increased to 30 nodes. The ratio of data channels to data flows was almost one to four (1:4). In any given time, there were about 4 data flows contending 
for 1 data channel. The amount of interference was high and the network resources were limited. The performance of CSA was superior and it improved as the size of the network was increased. It dropped fewer packets in each of the transmitting nodes. The number of dropped packets was less than the ones which were dropped in Fig 9. The results illustrate that the CSA scheme is more scalable and effective in addressing the MSC.

\subsection{Packet Drop Rate}

In these last set of results, the frequency at which the packets were dropped is analyzed. The reduction of the end-to-end delay shows that the proposed scheme is effective in addressing the MSC. It shows to what extent the system service rate and response time affect the behavior of the queue and the buffering of packets. If the service rate is poor, the queue builds up at a very faster rate, degrading the performance of the network. Lastly, it is a measure of how effective a system is in reducing the idle durations of channels and how it improves the utilization of the channels. In Fig 11, the effects of packet drop rate on system performance are analyzed in a network with 4 nodes. A system with shorter waiting times is desired as it suffers lower packet drop rates.

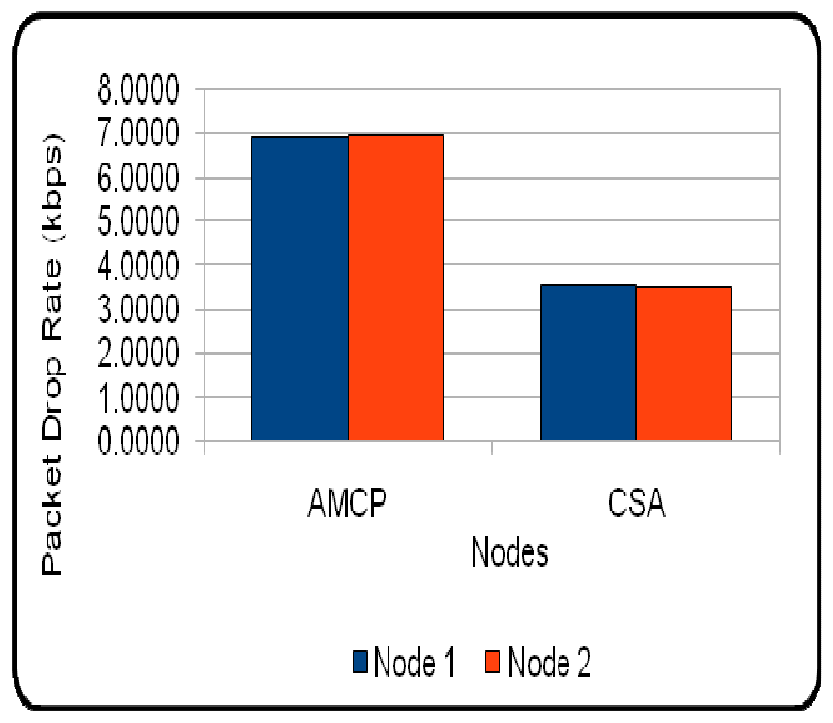

Fig 11. Impact of transmission delays in a network with 4 nodes

The transmission delays have a huge impact on the AMCP in Fig 11, and its packet drop rate is higher than that of the proposed scheme. The higher drop rate shows that the scheme is more prone to the MSC which result in the poor utilization of channels causing longer idle durations. The CSA had a lower packet drop rate owing to its ability to address the effects of the MSC. Its response time is superior resulting in lower packet drop rate, and shorter end-to-end delays.

In Fig 12, the CSA scheme also outperformed the AMCP. The network size was increased to 6 nodes to create a congested network state which is ideal for testing the scalability of protocols. The results demonstrate that the CSA is more scalable and more efficient in addressing the effects of the MSC. 


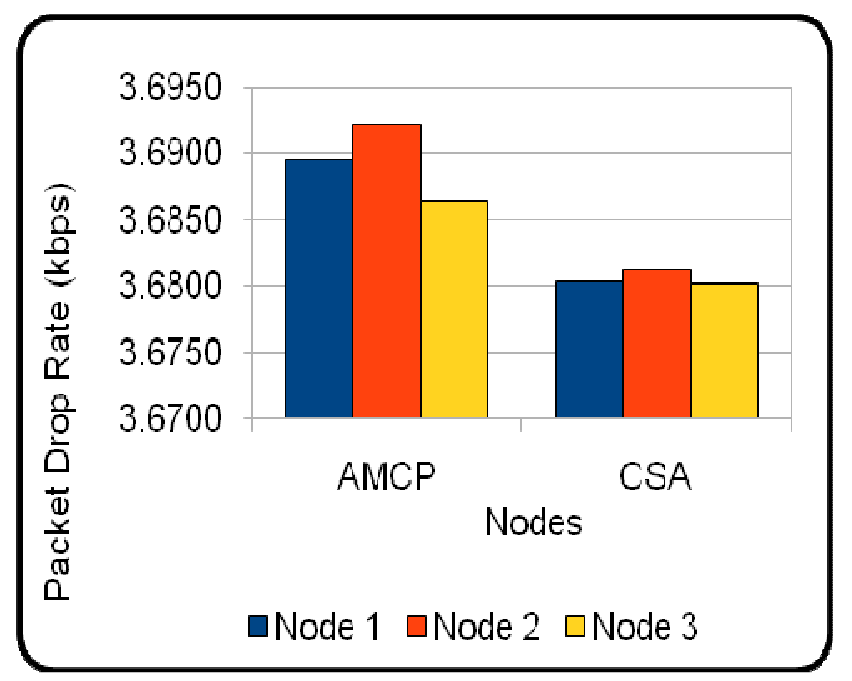

Fig 12. The analysis of the effect of MSC in a network with 6 nodes

Lastly, the size of the network was increased to 30 nodes in Fig 13. The data flows were almost 4 times the number of the channels resulting in a heavily congested and over loaded network. The CSA was superior and had lower packet drop rate in all but 2 nodes. It is superior, more scalable and effective in addressing the effects of the MSC. It improves the utilization of the channels, the system service rate, and the system response time furthermore, it also reduces the idle durations of the channels and the end-to-end delay.

The performance of the two schemes is poor in the central nodes largely due to the fact that as the inter arrival rate of packets increases, the buffers fill up at a faster rate while the congestion also increases steadily. When these to occurrences take places simultaneously, the performance of the network is degraded. However, performance thereafter improves with the passage of time when the schemes take advantage of spatial reuse. The TCP protocol also adjusts its transmission window when it detects congestion resulting in fewer packets being dropped. The superiority of the CSA is demonstrated by the fact that its drop rate is lower packets in comparison with that of the reference model.

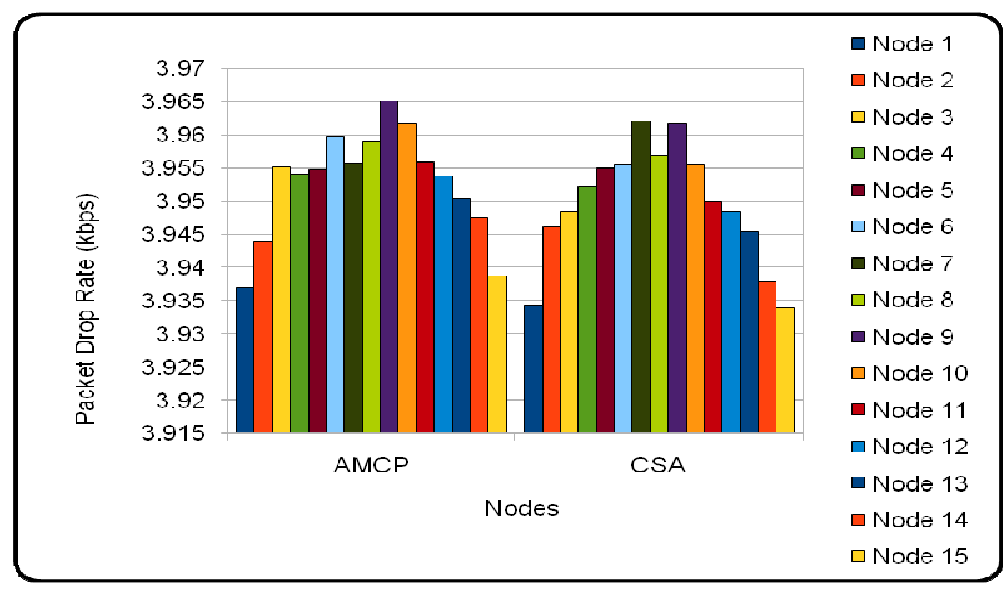

Fig 13. Evaluation of the CSA in a congested network 


\subsection{Conclusion}

The proposed CSA protocol reduces the MSC and limits it to the first cycle. It also reduces the idle durations of the channels and the signaling overhead cost. The system service rate and system response time are also improved which in turn improves the overall system performance. The results demonstrate that the CSA performs better in large and congested networks, which illustrate the scalability and the effectiveness properties of the scheme. In this case, a large network has 10 or more nodes. A distinguishable pattern was observed in large networks. The performance of the network worsens in the middle nodes before it improves again in the last nodes. This is caused by congestion and an increase in the data traffic however; it improves in the last nodes due to spatial reuse and the adjustment of the TCP window.

\section{References}

[1] R Maheshwari, H Gupta and S R. Das. "Multichannel MAC protocols for wireless networks". SECON 2006, Volume 2, Issue , 28-28 Sept. 2006.

[2] J So and N Vaidya (2004). "Multichannel MAC for Ad Hoc Networks: Handling Multichannel Hidden Terminals Using A Single Transceiver". MobiHoc 2004, Roppongi, Japan, ACM.

[3] J Chen and S Sheu. "Distributed Multichannel MAC protocol for IEEE 802.11 ad hoc wireless LANs". Computer Communications (2005). www.elsevier.com/locate/comcom

[4] H W So and J Walrand (2006). "Design of a Multichannel Medium Access Control Protocol for AdHoc Wireless Networks". University of California at Berkeley, EECS Department. http://www.eecs.berkeley.edu/Pubs/TechRpts/2006/EECS-2006-54.html

[5] W Chen, J Liu, T Huang, and Y Chang (2008). "TAMMAC: An Adaptive Multichannel MAC Protocol for MANETs". IEEE TRANSACTIONS ON WIRELESS COMMUNICATIONS, VOL. 7, NO. 11, NOVEMBER 2008

[6] A Muir and J.J. G Aceves.“A channel access protocol for multi hop wireless networks with multiple channels. University of California.’IEEE International Conference on Communications, 07 Jun 1998 -11 Jun 1998, Atlanta, GA, USA. http://ieeexplore.ieee.org/xpls/abs_all.jsp?arnumber=683102\&tag=1

[7] T Luo, M Motani, and V Srinivasan (2009). "Cooperative Asynchronous Multichannel MAC: Design, Analysis, and Implementation”. IEEE TRANSACTIONS ON MOBILE COMPUTING, VOL. 8, NO. 3, MARCH 2009

[8] A K Mahani, M Naderi, C Casetti, and C F. Chiasserini (2007). "ENHANCING CHANNEL UTILIZATION IN MESH NETWORKS”. IEEE MILCOM, Orlando, FL, 2007, 29-31 October 2007. www.ieeexplore.ieee.org/xpls/abs_all.jsp?arnumber=4454826

[9] J Nitin, S R. Das and A Nasipuri. "A multichannel CSMA MAC protocol with Receiver-Based Channel Selection for Multihop Wireless Networks.” Proceedings. Tenth International Conference on Computer Communications and Networks, 2001. 15 Oct 2001 - 17 Oct 2001, Scottsdale, AZ, USA. http://ieeexplore.ieee.org/xpls/abs_all.jsp?arnumber=956301

[10] J Shi, T Salonidis, and E W. Knightly (2006). "Starvation Mitigation Through Multichannel Coordination in CSMA Multi-hop Wireless Networks”, MobiHoc'06, May 22-25, 2006, Florence, Italy

[11] R Zhao and B Walke (2005). "Decentrally controlled wireless multi hop mesh networks for high quality multi media communications." MSWiM October 2005. http://portal.acm.org/citation.cfm?id=1089444.1089479 6 June 2007

[12] M Razfar and A Abedi (2011). "Single Channel Versus Multichannel MAC Protocols for Mobile Ad Hoc Networks." Proceedings of the World Congress on Engineering and Computer Science 2011 Vol II, WCECS 2011, October 19-21, 2011, San Francisco, USA

[13] C Shi1, H Zhao1, Y Tang, and Y Zhou (2012). "Analysis of the Multi-channel MAC Protocols in Wireless Networks." Proceedings of the 2012 2nd International Conference on Computer and Information Application (ICCIA 2012)

[14] S. M. KAMRUZZAMAN, Md. Abdul HAMID, M. Abdullah-Al-WADUD (2010). "An EnergyEfficient MAC Protocol for QoS Provisioning in Cognitive Radio Ad Hoc Networks." RADIOENGINEERING, VOL. 19, NO. 4, DECEMBER 2010 
[15] S. M. Kamruzzaman (2010). "An Energy Efficient Multichannel MAC Protocol for Cognitive Radio Ad Hoc Networks." International Journal of Communication Networks and Information Security (IJCNIS) Vol. 2, No. 2, August 2010

[16] H A Omar, W Zhuang and L Li (2011). "VeMAC: A Novel Multichannel MAC Protocol for Vehicular Ad Hoc Networks." IEEE MobiWorld 2011

[17] N Trivedi, G. Kuamr, T Raikwar (2013). "Survey on MAC Protocol for Wireless Sensor Network." International Journal of Emerging Technology and Advanced Engineering, Volume 3, Issue 2, February 2013

[18] L Tang, Y Sun, O Gurewitz, and D B. Johnson (2011). "EM-MAC: A Dynamic Multichannel EnergyEfficient MAC Protocol for Wireless Sensor Networks.” MobiHoc'11, May 16-19, 2011, Paris, France

[19] Md. A Hamid, M. Abdullah-Al-Wadud, and I Chong (2010). "A Schedule-based Multi-channel MAC Protocol for Wireless Sensor Networks.” Sensors 2010, 10, 9466-9480; doi:10.3390/s101009466, www.mdpi.com/journal/sensors, ISSN 1424-8220

[20] V Kumar and J Thakur (2013). "Multi-Channel Mac Protocols in Wireless Sensor Networks: A Review." International Journal of Advanced Research in Computer Science and Software Engineering, Volume 3, Issue 10, October 2013, ISSN: 2277 128X

[21] S Hsu, C Hsu, S Lin, and F Lin (2010). "The Analysis of a Self-stabilizing MAC Protocol for Multichannel Ad Hoc Networks.” JOURNAL OF NETWORKS, VOL. 5, NO. 8, AUGUST 2010

[22] K H. Almotairi and X (Sherman) Shen (2011). "Multichannel medium access control for ad hoc wireless networks." WIRELESS COMMUNICATIONS AND MOBILE COMPUTING Wirel. Commun. Mob. Comput. (2011)

[23] T Luo, M Motani, V Srinivasan." CAM-MAC: A Cooperative Asynchronous Multi-Channel MAC Protocol for Ad Hoc Networks" Broadband Communications, Networks and Systems, 2006. BROADNETS 2006

[24] J Nieminen and R Jäntti (2011). "Delay-throughput analysis of multi-channel MAC protocols in ad hoc networks." EURASIP Journal on Wireless Communications and Networking 2011

[25] B. S Kumar, P. SivaChandrika, A. S Kumar, and K. Srinivasulu (2013). "Energy - Effectual Strategies for Conjunctive Multichannel MAC Protocols." International Journal of Advanced Research in Computer and Communication Engineering Vol. 2, Issue 2, February 2013

[26] M Velempini and M. E. Dlodlo (2009). "Analyzing the effects of increasing data channels and the number of data flows on network performance." Wireless VITAE 2009, Aalborg, Denmark, 17-20 May, 2009.

[27] M Velempini and M. E. Dlodlo (2009). "A multiple channel selection and coordination MAC Scheme.” Mesh 2009, Glyfada/Anthens, Greece, 18 - 23 June 2009.

[28] E Jung and N H. Vaidya (2002). "A Power Control MAC Protocol for Ad Hoc Networks." MOBICOM'02, September 23-28, 2002, Atlanta, Georgia, USA.

[29] The editors of IEEE 802.11. Wireless LAN Medium Access Control (MAC) and Physical Layer (PHY) Specification, 1997. 